\title{
Die Rekonstruktion des Wimpergs über dem Westportal der Fürstenkapelle am Meißner Dom
}

\section{Günter Donath}

Rekonstruktionen haben in der Denkmalpflege bislang keinen guten Ruf. Aber ist diese starre Haltung auch gerechtfertigt? Natürlich ist das vordringliche Beschäftigungsobjekt der Denkmalpflege das tatsächlich noch vorhandene Denkmal. Auch ist sich der Verfasser darüber im Klaren, dass jede noch so gut dokumentierte und ausgeführte Rekonstruktion de facto ein Neubau ist, der das verlorene Original weder jemals vollgültig ersetzen noch Geschichte rückgängig machen kann. Aber in der baupflegerischen Praxis sind Rekonstruktionen - wie im Ergebnis eines am Meißner Dom 2011 durchgeführten wissenschaftlichen Kolloquiums zu konstatieren war - zum Wohle und Schutz der anvertrauten Monumente mitunter unumgänglich und an den europäischen Kathedralen nicht ungewöhnlich. Am Meißner Dom gibt es dazu mit der Wiederholung der Vermauerung der zur Mitte des 13. Jahrhunderts offenen Arkaden des Achteckbaus nach dem gut dokumentierten Vorbild der Zeit um 1370 bereits ein ausgeführtes Beispiel. Trotzdem bleibt die Erneuerung des schon seit über einhundert Jahren fehlenden Wimpergs ein Einzelfall - und für den planenden Architekten eine glückvolle Erfahrung beim notwendigen Repetieren des Wissens der mittelalterlichen Werkmeister im Planungsprozess.

\section{Was ist ein Wimperg?}

Wer sich mit der Baukunst der Gotik beschäftigt, wird rasch mit einer Vielzahl von Fachbegriffen konfrontiert. Während sich „Kriechblume“, „Kreuzblume“ oder „Maßwerk“ - „das gemessene Werk“ - fast von selbst erschließen, wird es bei einem „Wimperg" schon etwas schwieriger: das Wort kommt vom Althochdeutschen „wintperga“ und bezeichnet etwas, das vor Wind und Wetter schützt bzw. birgt. Ursprünglich verwendete man diesen Begriff für die Vorhallen vor den Portalen der Kathedralen, deren steile Satteldächer aufwändig gestaltete und verzierte Giebel zeigten. Im Laufe der Zeit ver-
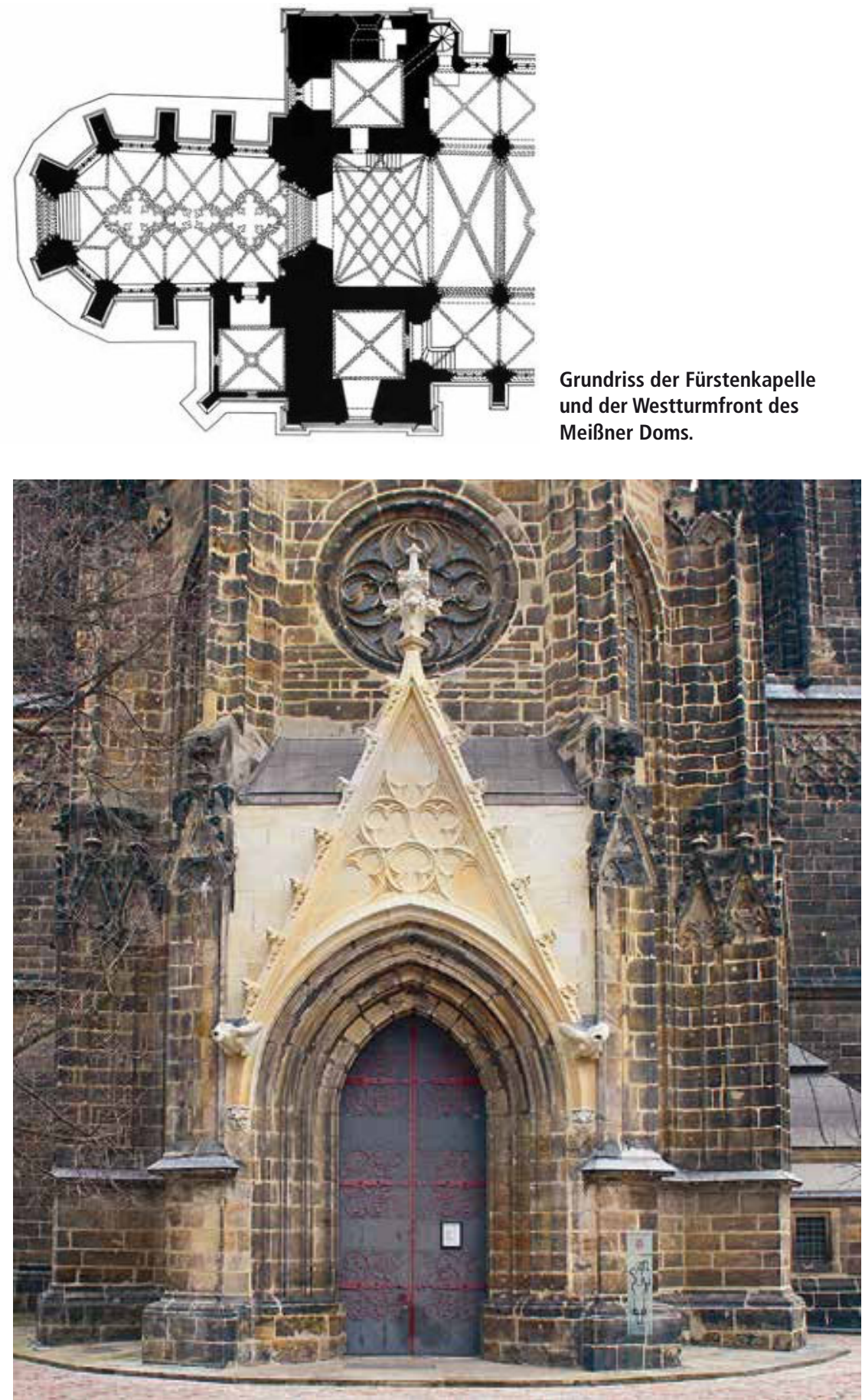

Dom zu Meißen, rekonstruierter Wimperg, 2015. 


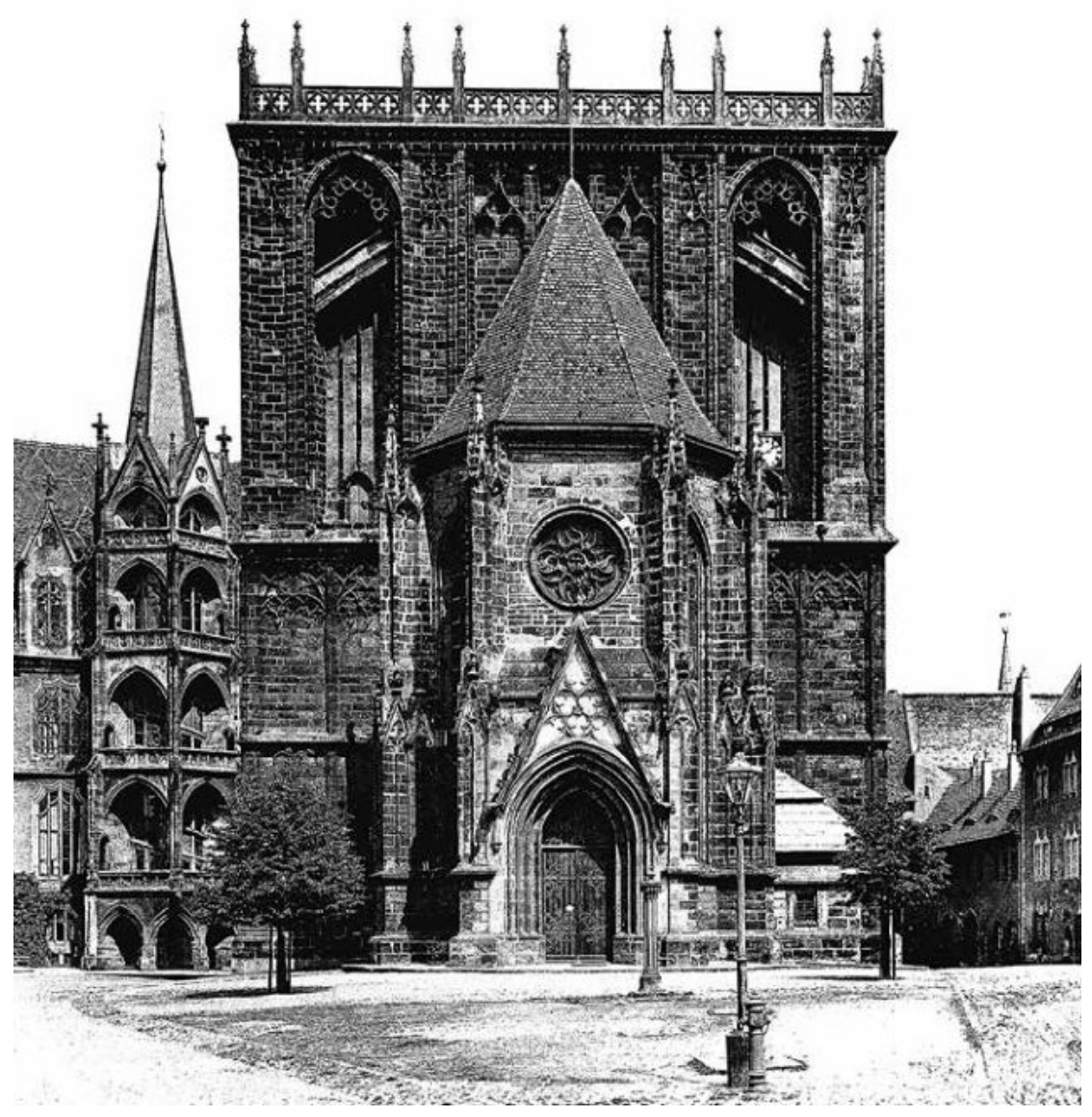

Die Westfassade des Meißner Domes, Messbildaufnahme von Albrecht Meydenbauer, 1898.

Westportal der Fürstenkapelle mit geschweiftem Dach des 17. Jahrhunderts, Lithographie nach einer Zeichnung von Gottlob Friedrich Thormeyer, um 1820.

1 Siehe auch Köpf, Hans und Binding, Günther: Bildwörterbuch der Architektur, Stuttgart 1999, S. 497.

2 Donath, Günter: Markgraf Wilhelm I. und der Meißner Dom als wettinisches Machtsymbol. In: Wilhelm der Einäugige, Markgraf von Meißen (1346-1407). Tagungsband.Schriften des Vereins für sächsische Landesgeschichte e. V., Bd. 11, Dresden 2009, S. 118-124.

\section{Zur Baugeschichte der Fürstenkapelle}

Das umgangssprachlich „Fürstenkapelle“ genannte Bauwerk wurde - kurz nach 1412 beginnend - wie ein Westchor dem Dom vorgelagert. Für die Errichtung der Begräbniskapelle ist offenbar die Allerheiligenkapelle östlich des Alten Palasts der Prager Burg, erbaut 1356 bis 1383 durch Peter Parler, von besonderer Bedeutung gewesen. Das Chorhaupt mit seiner fünfseitigen Brechung - wegen seines „Glashaus-Charakters" immer wieder mit französischen Architekturvorbildern in Zusammenhang gebracht - wurde zum baukünstlerischen Vorbild für zahlreiche mittelalterliche Chorfassaden um 1400, insbesondere der mitteldeutschen „schönen Chorfassaden“. Allerdings wurde die Prager Allerheiligenkapelle 1541 nach einem Brand stark beschädigt und danach in ihrem Inneren verändert. Ist aber das Formprinzip der Prager Allerheiligenkapelle ermittelt, so fällt es nicht schwer, es auch im Bau der Meißner Fürstenkapelle zu entdecken; insbesondere in den Verschlingungen und Kleinformen der Strebepfeilerarchitektur. Die große Ähnlichkeit dieser beiden Bauten verblüfft immer wieder, insbesondere auch beim Vergleich der ursprünglich geplanten Gewölbe mit dem Parallelrippenschema, welches Peter Parler bereits im Chor von St. Veit geschaffen hatte.

Auftraggeber des Baus war Markgraf Friedrich der Streitbare (1370-1428), der sich bei der Teilung der wettinischen Lande nach dem Tode Markgraf Wilhelms I. (1343- 1407), des Einäugigen, nach der Leipziger Örterung für die Mark Meißen entschieden hatte. Friedrichs Bruder, Wilhelm II. (1371-1425), hatte zuvor schon am Altenburger Schloss ein Stift gegründet und dort begonnen, einen Chorbau zu errichten. Gleiches beabsichtigte nun Friedrich der Streitbare, der den Meißner Burgberg mit Schloss und Dom stärker zum Zentrum seiner Landesherrschaft ausbauen wollte. Bereits Wilhelm I. hatte den Meißner Dom als Kathedrale eines von ihm neu begründeten Landesbistums und als Ort seiner Grablegung auserkoren. Deshalb trieb er die Vollendung des Dombaus voran. 1401 erfolgte die Schlussweihe des Domes.

Allerdings waren mit der Bestattung Wilhelms I. und seiner Gemahlin Elisabeth von Mähren im so genannten Stifterjoch des Hohen Chors des Domes, zu Füßen der Standbilder des Kaiserpaars, die prominentesten Plätze belegt. ${ }^{2}$ Um einen standesgemäßen Ort für dynastisches Gedenken, der Fürbitte und des Begräbnisses $\mathrm{zu}$ erhalten, ließ Friedrich vor dem 
Westportal des Meißner Doms einen neuen Westchor errichten. Mit einer inneren Breite von knapp $9 \mathrm{~m}$ und 16,9 m Länge wurde der Kapellenneubau dem breiten Westturmriegel des Domes vorgelagert. Für den Grundriss wählte man ein im Westen im 5/8-Schluss endendes Polygon. Offenbar merkte man bald nach der Absteckung, dass das zwischen den eng gestellten Polygonpfeilern angeordnete neue Portal zu schmal ausfallen würde. Deshalb verbreiterte man dessen lichte Weite und war nun gezwungen, die Nordwand der Kapelle von der geplanten Parallelstellung zur Südwand um ca. $60 \mathrm{~cm}$ abweichend leicht nach außen zu führen, sodass sich im Westen der Kapelle eine komfortable Breite für das neue Portal ergab. Außen erfährt der Meißner Bau in der Verdichtung der Schmuckformen eine Steigerung von Ost nach West. Die Strebepfeilerflanken sind mit wimpergartigen Blendmaßwerken reich verziert. In der Achse der Westfassade, am Ort eines hierarchisch wichtigen Portals, das noch einmal durch eine hoch oben angesetzte Fensterrose betont wird, befindet sich das Portal. Es widerspräche völlig den gestalterischen Prinzipien der „schönen Chorfassaden“, wenn dieses ganz schmucklos ohne Wimperg geblieben wäre.

1428 wurde Friedrich der Streitbare (13701428), nunmehr Kurfürst von Sachsen, inmitten der neuen Fürstenkapelle beigesetzt. Der liturgische Dienst der für die Fürstenkapelle zuständigen Priester - die Klerikergemeinschaft in Dom und Fürstenkapelle hatten um 1480 einen „ewigen Chor" etabliert - endete 1539 mit Einführung der Reformation. Nach Vernachlässigung und willkürlichen Beschädigungen im Dreißigjährigen Krieg wies Kurfürst Johann Georg II. (1613-1680) eine Restaurierung der Fürstenkapelle an. Von 1662 bis 1676 erfolgte deshalb eine barocke Umgestaltung der Kapelle durch Oberlandbaumeister Wolf Caspar von Klengel (16301691), der im Geiste seiner neuen Stilauffassung das Portal nun mit einer kleinen Vorhalle versah und diese mit einer Schweifhaube überdachte. Prof. Dr. Bruno Klein (TU Dresden) vermutet, dass sich deren etwas ungewöhnliche Gestalt des Schweifdaches damit erklären lässt, dass mit dieser Dachgestalt Rücksicht auf den noch bestehenden Wimperg oder zumindest auf Teile von ihm genommen wurde. ${ }^{3}$

\section{Der Wimperg von 1865 und seine Beseitigung}

Gleichzeitig mit der Wiederentdeckung der gotischen Baukunst im 19. Jahrhundert setzte die Beschäftigung und Auseinandersetzung mit dem Meißner Dom ein. Die Maler der Romantik, unter ihnen Philipp Otto Runge (1777-1810) und Johann Theodor Goldstein (1798-1871), ein Schüler Caspar David Friedrichs (1774-1840), nahmen in ihren gezeichneten Visionen die später erfolgte Vollendung des Domes vorweg. Die Fürstenkapelle bildeten sie ohne die barocken Veränderungen $a b$ und malten einen gotischen Wimperg über dem Portal.

Nach langwierigen Vorbereitungen begann 1856 die erste umfassende Restaurierung des Meißner Doms. Die Arbeiten wurden von König Johann (1802-1873) gefördert und ein Schüler Gottfried Sempers (1803-1879), der Architekt Christian Friedrich Arnold (18231890), übernahm als Dombaumeister die Bauplanung. 1865 stellte Arnold den alten Zustand

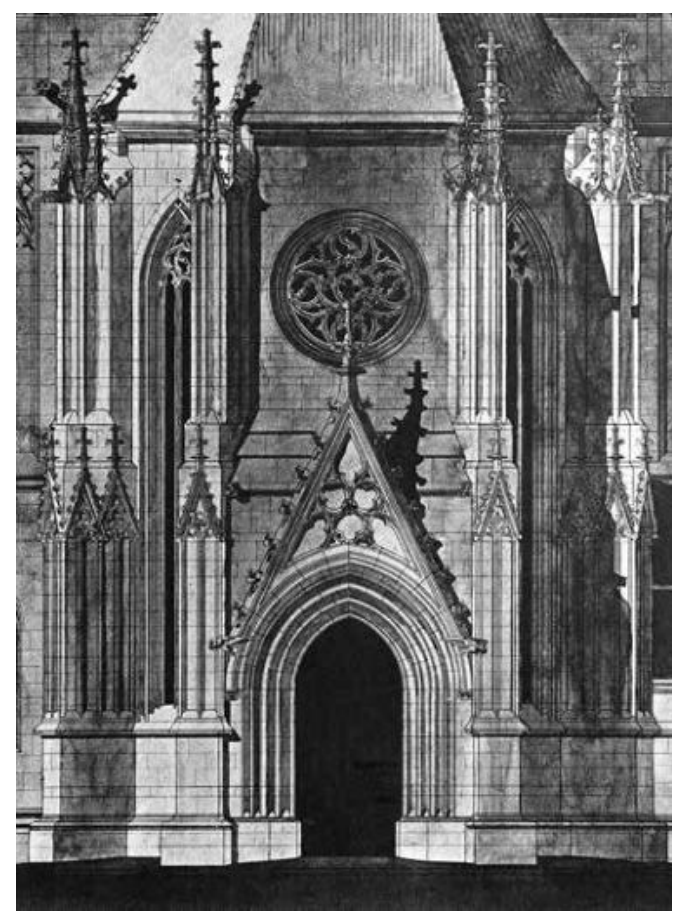

am Westportal der Fürstenkapelle wieder her, indem er den Wimperg über der Westtür wieder errichten ließ. ${ }^{4}$ Seine Architekturformulierungen und Detailausbildungen sind natürlich Interpretationen gotischer Architektur, aber als solche nicht weit entfernt von der Stilhaltung der „schönen Chorfassaden“.

Doch Arnolds faszinierendes Werk war nur von kurzer Dauer. Während Dombaumeister Carl Schäfer (1884-1908) bei den Entwürfen für den Ausbau der Westtürme des Domes die Existenz des Wimpergs nie in Frage stellte und ihn ganz selbstverständlich in die Gesamtkonzeption der nach oben strebenden Hauptachse der Mittelpartie der Westansicht einbezog, leg-

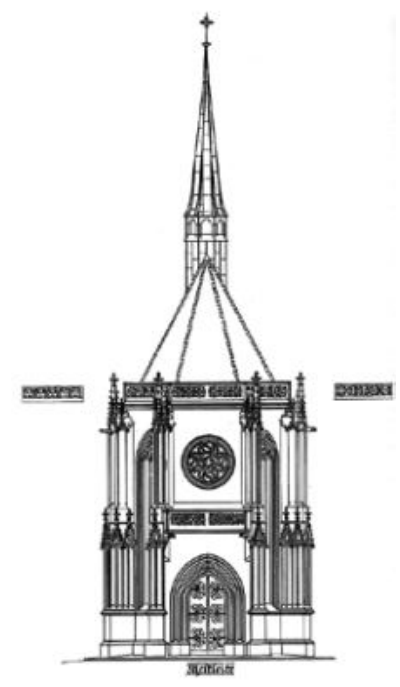

Meißner Dom, Westansicht der Fürstenkapelle mit Maßwerkbalustraden über dem Portal sowie über dem Dachansatz, Entwurf, 1908.

Westansicht des Meißner Domes, Zeichnung von Carl Schäfer, Ausschnitt mit dem Westportal an der Fürstenkapelle und dem Wimperg von 1865, um 1904

\footnotetext{
3 Klein, Bruno: bauhistorische und denkmalpflegerische Überlegungen zur Wiedererrichtung des Wimpergs über dem Westportal der Fürstenkapelle, in: Monumenta Misnensia, Jahrbuch für Dom und Albrechtsburg zu Meißen 11 (2013/2014), S. $49-51$.

4 Datierungen nach Matthias Donath, in: Donath, Günter (Hrsg.): Die Restaurierung des Doms zu Meißen 1990-2002. Stuttgart 2003, S. 30-36.
} 


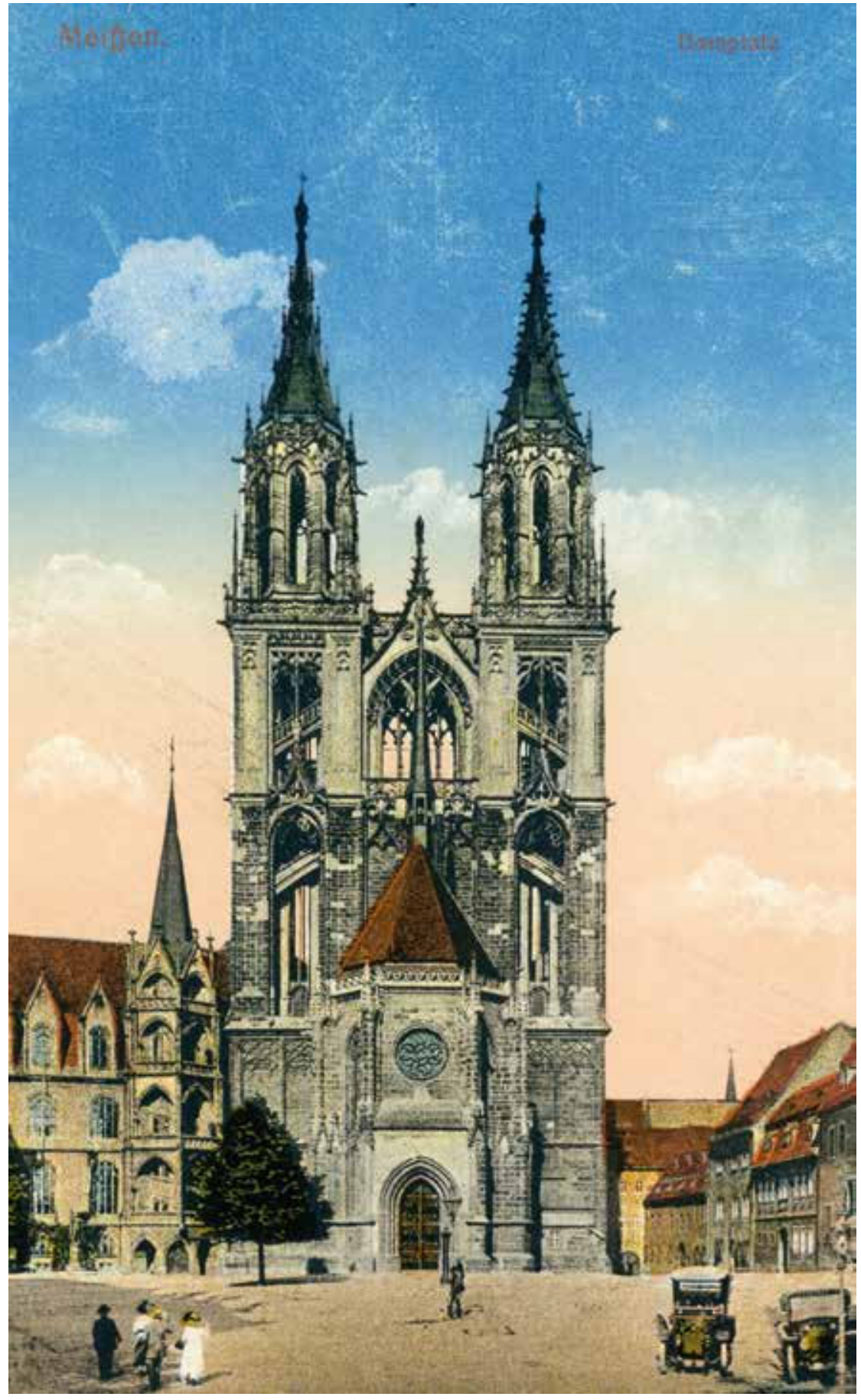

Meißner Dom, Westansicht, Postkarte um 1910.

5 Alle Angaben stammen aus den Bauakten im Büro des Dombaumeisters/ Hochstift Meißen.

6 Magirius, Heinrich: Stil und Stilkorrekturen in der Denkmalpflege seit 1900. Annäherungen an das rechte $\mathrm{Maß}$, in: Monumenta Misnensia, Jahrbuch für Dom und Albrechtsburg zu Meißen 11 (2013/2014), S. 43 . tung über dem Traufgesims und den Bau eines Dachreiters vor. In einer Stellungnahme der Königlichen Kommission zur Erhaltung der Kunstdenkmäler vom 22. September 1908 wurde gegen diese Maßnahmen, aber für den Dachreiter entschieden. In einem weiteren Gutachten vom 14. Oktober 1908 - der Ausbau der neuen Westtürme war nahezu vollendet sprach sich die Kommission sogar für die Erhaltung des Wimpergs aus. Allerdings hatte Hartung mit seinen Absichten einen erklärten Feind der Neugotik an seiner Seite: Cornelius Gurlitt (1850-1938). Diesem gelang es, die Kommission umzustimmen und teilte im Januar 1909 mit: „Mit dem Vorschlage, die häßliche Bekrönung des Portals verschwinden zu lassen, ist die Kommission einverstanden. Nur findet sie, daß sich durch Neueinfügung einer Maßwerkgalerie dort ein Ablagerungspunkt [...] ergeben werde."6

Um seine vorrangige Idee, die Fürstenkapelle mit einer Maßwerkbrüstung $\mathrm{zu}$ bekrönen, letztendlich durchzubringen, „opferte“ Hartung die Galerie über dem Portal und versah diese Stelle mit einer pultdachartigen Konstruktion aus Sandstein. Die unmittelbar nach der Turmvollendung entstandenen frühesten Fotografien der Westseite des Domes geben diese Situation anschaulich wieder: Auf der

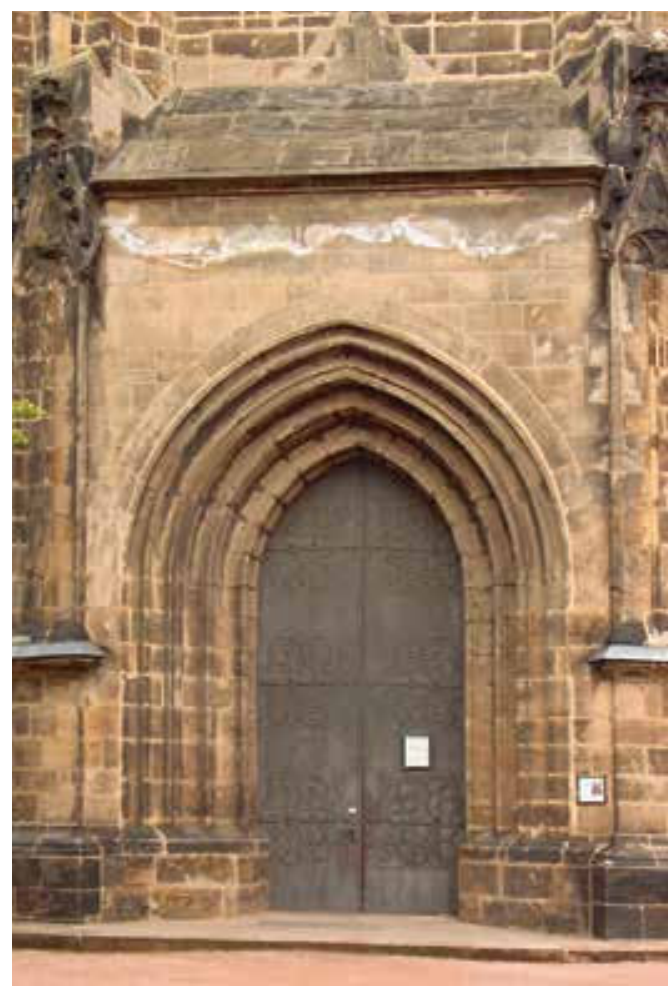

Westportal der Fürstenkapelle am Meißner Dom mit Salzausblühungen unterhalb des Traufgesimses, Ansicht vom Domplatz, Zustand 2005. 
Postkarte von 1908 sieht man die neuen Domtürme und das Portal mit dem Wimperg. Die Postkarte von 1909 zeigt an der Stelle des Wimpergs nun das steinerne Pultdach und die beiderseits der Portalarchivolte ebenfalls neu in Sandstein aufgeführten Wandflächen als hellen Fleck.

\section{Vorüberlegungen zur Rekonstruk- tion des Wimpergs}

Die Wasserableitung an dem Pultdach war allerdings nicht so einfach, wie es sich Hugo Hartung gedacht hatte. Vielmehr bot diese Schräge geradezu ein Einfallstor für eindringende Feuchtigkeit. Unter der Schräge zeichneten sich herablaufende Wasserfahnen und weiße Salzablagerungen ab.

Im Zusammenhang mit den umfangreichen steintechnischen Reparaturarbeiten an der Fürstenkapelle in den Jahren 1997 bis 2001 konnten zwar die geöffneten Fugen in der Pultdachschräge immer wieder einigermaßen regendicht verschlossen werden, jedoch blieben die offensichtlichen Steinschäden der technisch unbefriedigenden Portallösung ein Pflegefall, der immer wieder Gerüststellungen erforderlich machte. 2006 wurden im Inneren der Kapelle in einem Pilotprojekt die beiden spätgotischen und für den mitteleuropäischen Kunstraum wegen ihrer Größe einzigartigen Terrakottaskulpturen restauriert. Da sie unmittelbar am inneren Portaltrichter aufgestellt waren, betraf sie die Gefährdung durch eindringende Feuchtigkeit und auch durch die im Inneren feststellbaren Salzablagerungen unmittelbar. Es galt nun, eine Lösung zu finden, die einen permanenten und nicht durch Reparaturzyklen bestimmten Schutz versprach. Das Abdecken der wasserbelasteten Steinschichten mit Blei wäre eine technisch einfach machbare Lösung gewesen. An vielen vorspringenden Gesimsen, auf der Aussichtsplattform der Westturmanlage oder auf dem äußeren Umgang des Hochchores hat sich diese Methode der Abdichtung bestens bewährt. Aber wäre sie auch den der gotischen Architektur innewohnenden Gesetzmäßigkeiten an der Portalfront gerecht geworden? So ein unprofilierter seitlicher Anschluss und pultdachartiger Übergang zur Fassade oberhalb eines Portals kommt in gotischen Architekturformulierungen überhaupt nicht vor. Man sah dieser Lösung an, dass sie durch bauliche Veränderungen einer anders geformten Portalzone entstanden war. Sowohl stilistisch als auch ästhetisch stellte sie ein unbefriedigendes Provisorium dar. Diese Notlösung konterkarierte zudem die Absicht der ehemals hier befindlichen Portalbekrönung, auf den Eingang zur Fürstenkapelle hinzuweisen.

Vor die Aufgabe gestellt, dieses Provisorium erhalten und konservieren zu müssen, begannen 2006 die ersten konkreteren Überlegungen, die baukonstruktiven und architektonischen Probleme an der Westtür der Fürstenkapelle durch die Wiedererrichtung eines Wimpergs zu lösen. Am 7. November 2011 führte der Dombau-Verein Meißen ein wissenschaftliches Kolloquium durch, zu dem Aspekte der Bau- und Kunstgeschichte sowie der Bedeutung von Rekonstruktionen in der Denkmalpflege diskutiert wurden. Die beteiligten Denkmalpfleger, Dombaumeister und Architekturhistoriker, unter ihnen die Kölner Dombaumeisterin Prof. Dr. Barbara Schock-Werner, sprachen sich für die Idee aus, den Wim-
Messnetz für die händische Bauaufnahme am gotischen Portal der Fürstenkapelle zur Überprüfung der geometrischen Unregelmäßigkeiten in der Linienführung der Portalarchitektur, 2014.

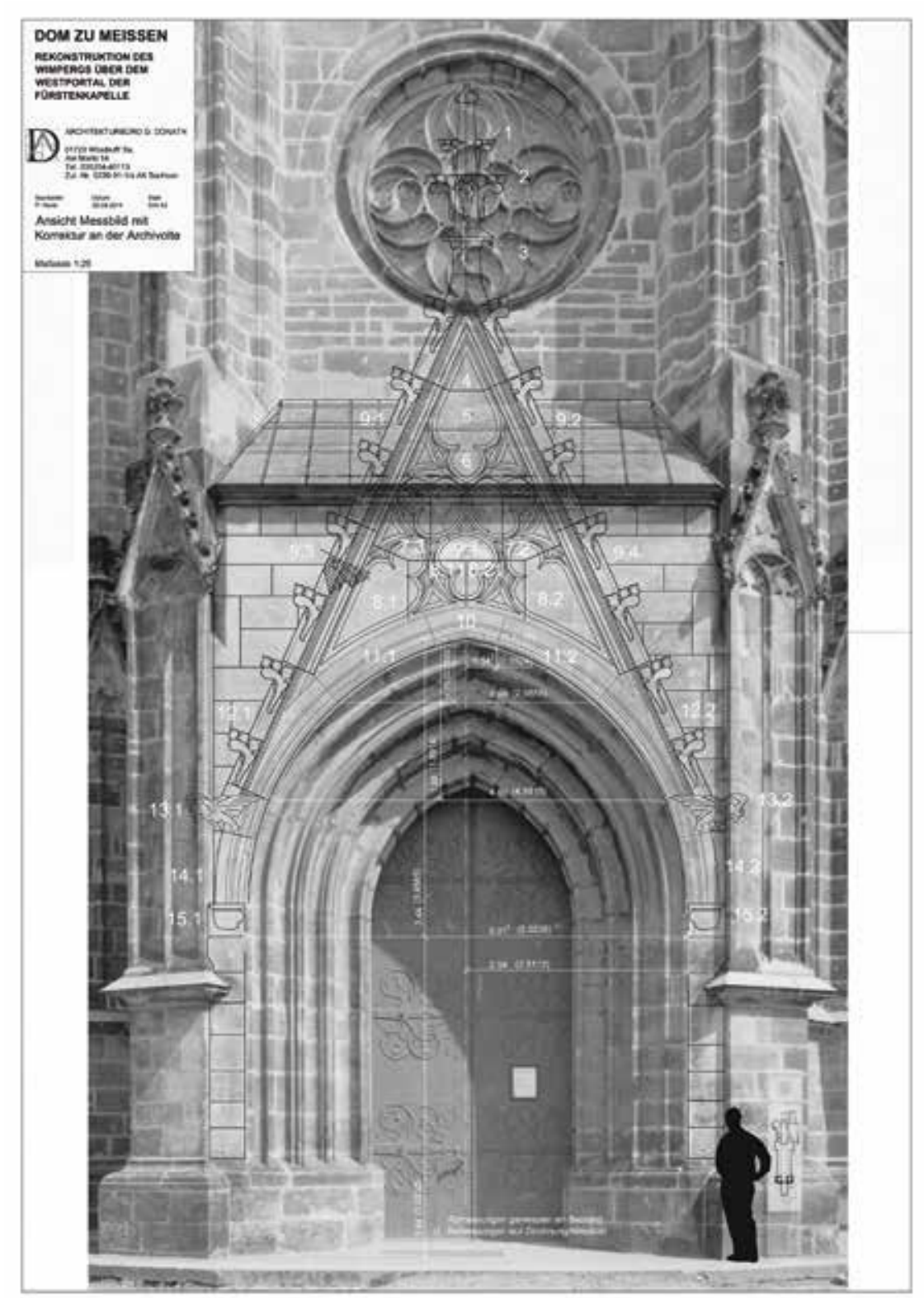


Werksteinübersicht mit Verteilung der Steinmetz- und Bildhauerarbeiten, 2014.

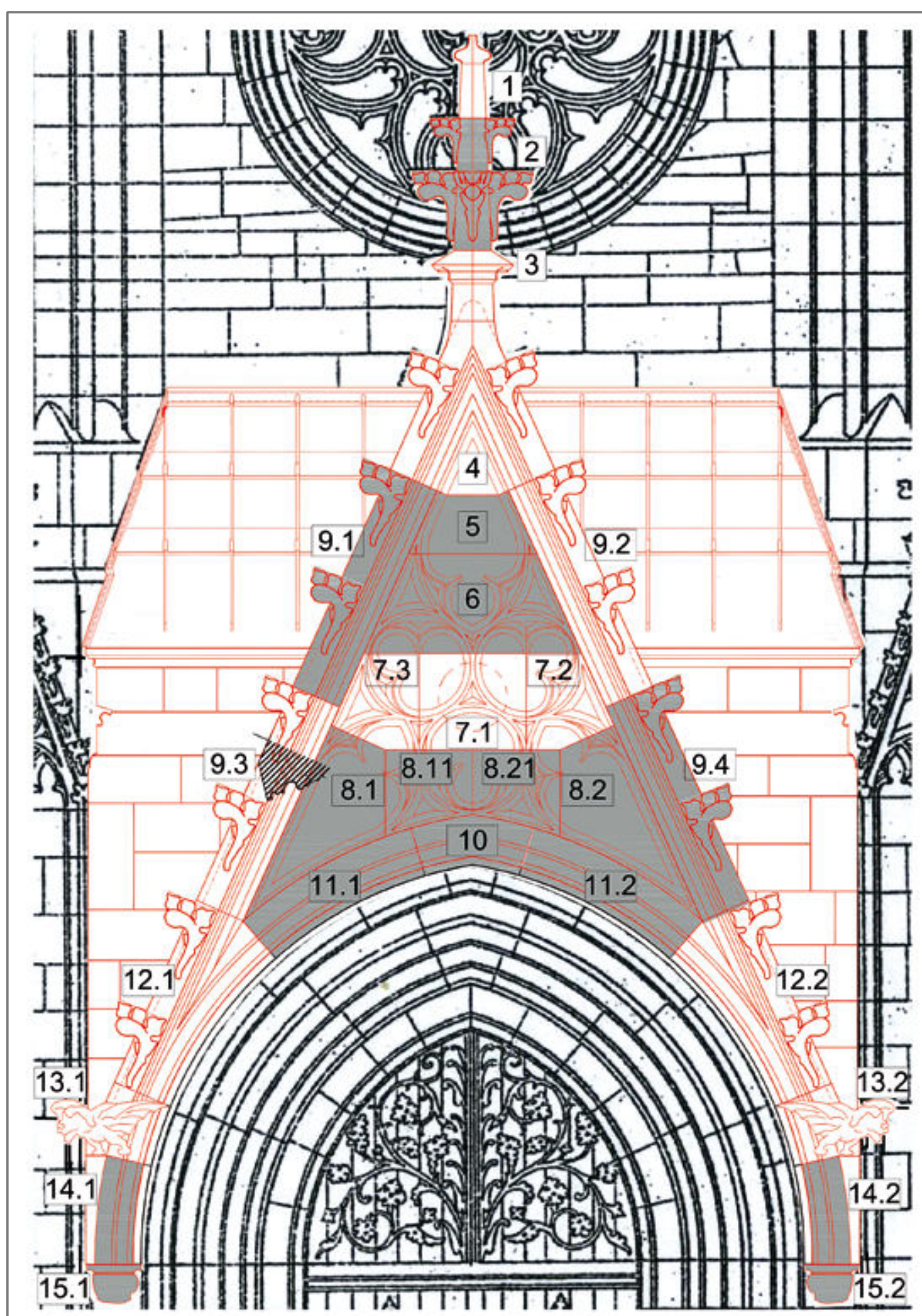

WERKSTEINE

2 Kreuzblume: Uwe Müller, Steinrestaurierung Neubert, Halsbrücke

5 oberes Giebelstück: Jonny Franke, Naturstein Tauscher, Lichtenstein

6 Giebelstück, Blendmaßwerk, Fischblase und Dreipässe : Sebastian Geier, Weimar

8.1/8.2 li. und re. Teil, Fischblase: Clemens Modrakowski, Dresden-Pillnitz

8.11/8.12 li. und re. Teil, Blendmaßwerk: Clemens Modrakowski, Dresden-Pillnitz

9.1 Ortgang mit Kriechblumen: Benjamin Hartmann, Fuchs+Gierke, Ottendorf-Okrilla

9.2 Ortgang mit Kriechblumen: Bohumil Panek, Prag

10 Schlussstein: Ansgar Julian Kamphenkel, Steinrestaurierung Neubert, Halsbrücke

11.1/11.2 Archivolte: Steinrestaurierung Neubert, Halsbrücke

14.1/14.2 Kämpfer: Tim Schliephake, Bauhütte Naumburg

15.1/15.2 Laubwerkkonsol: Meret Gerber (Wandergesellin, Schweiz) 


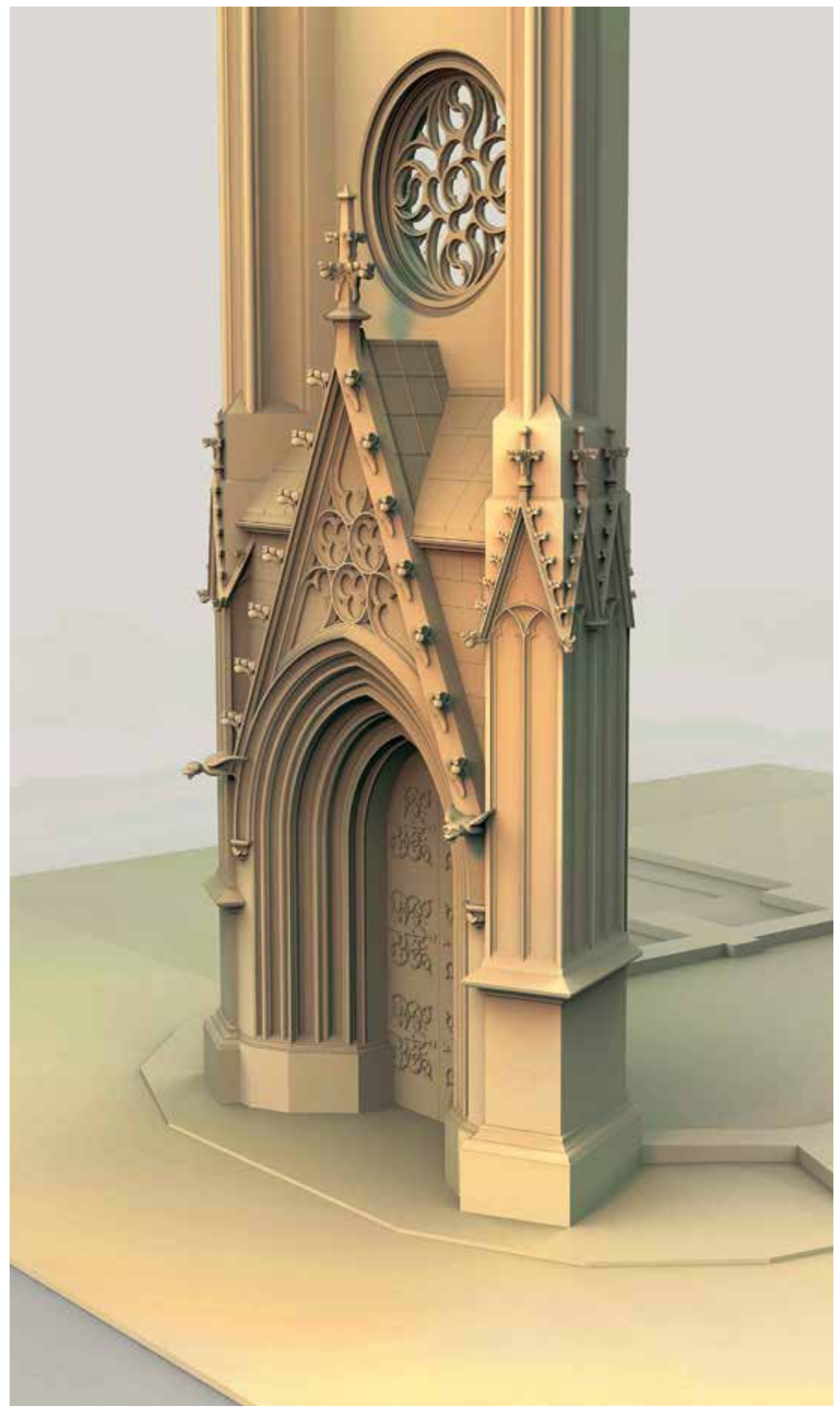

Dom zu Meißen, Rekonstruktion des Wimpergs über dem Westportal der Fürstenkapelle. 3-D-Darstellung: Christine Fuhrmann und Christoph Bethlehem, Halle/Saale 
perg in der Gestalt der 1860er Jahre wiederzuerrichten. Domherr Prof. Dr. Heinrich Magirius und Prof. Dr. Bruno Klein begleiteten die weiteren Planungen aus denkmalpflegerischer und bauhistorischer Sicht.

\section{Bauplanung und Rekonstruktion}

Ein wesentliches Argument für die Rekonstruktion war die Tatsache, dass der 1909/10 beseitigte Wimperg außerordentlich gut dokumentiert war. 1898 hatte der Vermessungsingenieur Albrecht Meydenbauer (1834-1921) den gesamten Dom innen und außen fotogrammetrisch aufgenommen und vermessen. Der Zeichner Johannes Unte hatte auf der Grundlage dieser Vermessung steingenau gezeichnete Ansichten und Gebäudeschnitte angefertigt. Damit war eine Voraussetzung für die getreue Wiederherstellung des Wimpergs erfüllt. Die Planung und Vorbereitung der Maßnahme nahm fünf Jahre in Anspruch. So musste die Finanzierung gesichert werden. Der DombauVerein Meißen erklärte die Wimperg-Rekonstruktion zu seinem vorrangigen Vorhaben, finanzierte die Voruntersuchungen und stellte einen finanziellen Grundstock bereit, dem dann noch Mittel der Ostdeutschen Sparkassenstiftung, der Sparkasse Meißen und der Deutschen Bundesstiftung Umwelt hinzugefügt werden konnten. Daneben mussten die Planung vertieft sowie Kosten- und Materialüberschläge angestellt werden. Eine 3-D-Visualisierung brachte wichtige Erkenntnisse zur räumlichen Zuordnung der freistehenden Giebelebene vor der Kapellenfassade.

Um zu überprüfen, ob noch Befunde des ursprünglichen Wimpergs aus dem 15. Jahrhundert oder des Wimpergs von 1865 vorhanden sind, wurde das Westportal 2011 eingerüstet und einer bauarchäologischen Untersuchung unterzogen. Dazu wurden einige der Sandsteinplatten der von Dombaumeister Hugo Hartung angeordneten Verdachung aufgenommen, um hinter die Konstruktion von 1909/10 zu schauen. Mit den bauarchäologischen Untersuchungen konnten Materialanalysen verbunden werden, die vom Institut für Diagnostik und Konservierung an Denkmalen in Sachsen-Anhalt und Sachsen (IDK) durchgeführt wurden. Zusätzlich zu der geöffneten Schräge stand Material aus zwei Kernbohrungen zur Verfügung. Die Untersuchungen ergaben, dass Teile einer älteren Konstruktion hinter bzw. unter dem Steinwerk von 1909/10 vorhanden waren, dass aber bei der Einfügung dieses Steinwerks der ältere Bestand größtenteils zerstört worden war. Die 1909 ausgeführte
Steinkonstruktion lag oberhalb und vor der Vorderkante der historischen Baufront und verdeckte somit seitlich das Blendmaßwerk der Strebepfeilerflanken. Die Mörtelanalysen belegten im Vergleich mit den im Inneren der Fürstenkapelle aus ungestörten Bereichen entnommenen Referenzmörteln, dass die mit ihnen verhafteten Bauteile zum Ursprungsbau gehörten. Wenn an Hand der Befunde auch nicht das Bild des gotischen Wimpergs zurück gewonnen werden konnte, so bestätigten die baulichen Reste zumindest, dass es einen solchen auf jeden Fall gegeben hat.

Nachdem die Finanzierung des Vorhabens gesichert war, konnte im Frühjahr 2014 mit der Umsetzung begonnen werden. Nun mussten für die Werkplanung genauere Vermessungen angefertigt werden. Dazu sollten sowohl händische Aufmaße als auch neu angefertigte maßstäblich entzerrte Messbildaufnahmen der gesamten Fassade bis hinauf zur Fensterrose dienen. Erst durch die Überlagerung beider Messergebnisse konnten maßliche Ungenauigkeiten ausgeräumt werden und der WimpergAufriss gezeichnet werden. Als Messpunkte am Bau dienten in Spreizdübel eingesetzte Edelstahlnägel, die mit Fäden verspannt wurden. Das so gewonnene Mess-Netz konnte über die gesamte Bauzeit hinweg für maßliche Kontrollen genutzt werden. Für die Geometrie des neuen Wimpergs wurden die Vergleichsmaße aus der Meydenbauer-Photographie und der Umzeichnung immer wieder abgeglichen und in das Messbild übertragen.

Die Visualisierung der innigen Verzahnung zwischen der vorhandenen Portallösung und dem Wimperggiebel konnte nur durch eine dreidimensionale Darstellung gelöst werden. Wichtig war dabei die bei der bauarchäologischen Untersuchung gewonnene Erkenntnis, dass die Vorderfront der neuen, den Wimperggiebel flankierenden Flächen etwa $19 \mathrm{~cm}$ hinter der aktuellen Vorderkante des Bestandsquaderwerkes liegt. Das Bestandsmauerwerk war deshalb so weit zurückzuarbeiten, dass der neue Wimperg wieder exakt in der originalen geometrischen Position aufgebaut werden konnte und eine konstruktiv sichere Verzahnung mit dem dahinter liegenden Mauerwerkskern der gotischen Wandkonstruktion gewährleistet war. Gleichzeitig mit dem Entstehen von Vertikal- und Horizontalschnitten mussten über die unterschiedlichen Einbindetiefen der Werksteine des neuen Wimpergs in das Bestandsmauerwerk die Rohblockgrößen für jeden einzelnen Werkstein festgelegt werden. 
Zuvor musste aber die Werkzeichnung des Wimpergs im Maßstab 1:1 ausgedruckt, auf dem Reißboden ausgelegt und mit der ebenfalls auf den Maßstab 1:1 vergrößerten Umzeichnung von Johannes Unte (1898) verglichen werden. Das gestaltete sich insofern schwierig, da bei der um das 100 -fache vergrößerten Zeichnung natürlich auch deren Strichstärken entsprechend vergrößert wurden und somit je nach Linienstärke im Original 1 bis 2 $\mathrm{cm}$ dicke Balken abgebildet wurden. Über die so vergrößerte Zeichnung wurde nun der auf Transparentpapier 1:1-Aufriss des neuen Wimpergs aufgelegt und die Linienführung aufeinander abgestimmt. Neben der starken Vergrößerung der Linien erschwerte auch die Tatsache den Abgleich, dass Unte den Wimperg-Aufriss nicht exakt konstruiert sondern der Messbildfotografie lediglich mehr oder weniger graphisch exakt nachempfunden hatte. Korrigiert wurde deshalb dabei nicht nur der Steinschnitt, sondern - in Verbindung mit der Herstellung der im Steinmetzbetrieb benötigten 1:1-Werksteinschablonen - auch die geometrische Durchbildung des Maßwerks. Diese Aufgabe wurde vor allem gemeinsam mit dem Bildhauer der Dombauhütte gelöst. Alle Korrekturen wurden in einen neuen Werksteinplan übertragen, der von nun an die Ausführungsgrundlage darstellte.

\section{Auswahl des Sandsteinmaterials}

Die steintechnischen Arbeiten des Dombaumeisters Friedrich Arnold waren stets dadurch gekennzeichnet, dass dieser offenbar eine Vorliebe für einen gelblichen Sandstein hatte, der in seiner Verwitterungsbeständigkeit deutlich hinter dem hochfesten quarzitisch gebundenen Sandstein der mittelalterlichen Bauabschnitte zurückblieb. Auch der Wimperg von 1865 bestand aus einem solchen Stein. Bereits beim Bau der Fürstenkapelle um 1415 hatten die Werkmeister ebenfalls einen gelben, grobkörnigen Sandstein der Postaer Varietät verwendet. Es lag deshalb nahe, diesen oder ähnliches Steinmaterial bei der Wimperg-Rekonstruktion einzusetzen. Bei Steinbruch-Begehungen kam man zu dem Entschluss, auch wegen seiner lebendigen Bänderung und der gelben, dem Originalgestein der Fürstenkapelle sehr nahe kommenden Färbung einen gelben Reinhardtsdorfer Sandstein zu verwenden. Mit den Sächsischen Sandsteinwerken Pirna war rasch ein Partner gefunden, der die für den Bau benötigte Steinmenge von 13,9 $\mathrm{m}^{3}$ (mehr als 33 t Stein!) in sechsseitig diamantgesägten Qua-

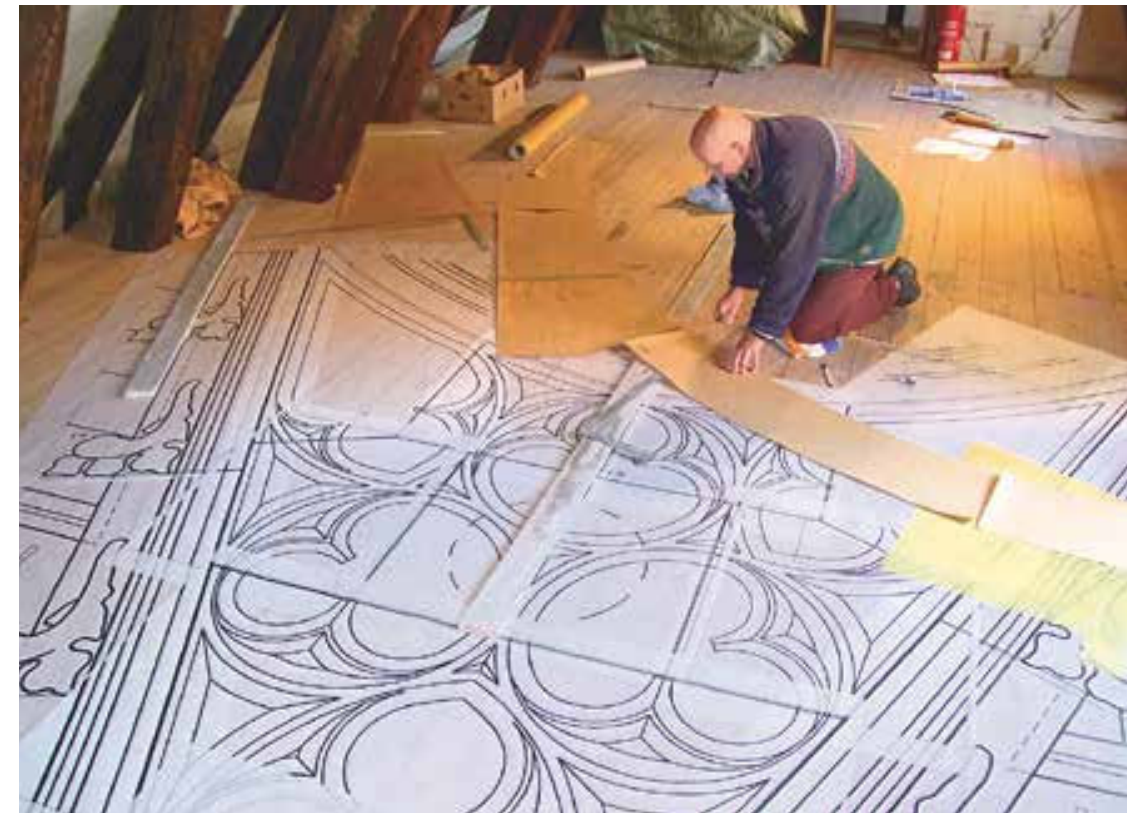

dern $\mathrm{zu}$ einem vergleichsweise moderaten Preis liefern konnte.

Bei der Bearbeitung der Steine bezog man von Anfang Auszubildende von Steinmetzschulen in die Durchführung dieser in der alltäglichen Baupraxis doch äußerst selten vorkommenden Bauaufgabe ein. So konnten über die Steinmetzschule in Demitz-Thumitz fünf Werksteine als Gesellenstücke und ein Meisterstück an ausgewählte Mitarbeiter renommierter Steinmetzbetriebe vergeben werden. Dazu gab es noch Bewerbungen freiberuflicher Steinmetze. Außerdem wurde die Steinmetzschule in Hořice in Nordböhmen einbezogen, die u.a. auch am Prager Veitsdom tätig ist. Alles andere fertigte die Dombauhütte Meißen an. Ihr oblag auch der Bau der 1:1-Gipsmodelle als bildhau-

Herstellung von 1:1-Werksteinschablonen. Überlagerung der auf Maßstab 1:1 vergrößerten UnteZeichnung mit der im Maßstab 1:1 auf Transparentfolie ausgedruckten Computerzeichnung der Werksteine des Wimperg-Aufrisses für die Herstellung der Werksteinschablonen auf dem Reißboden, 2014

Steinmetzarbeiten vor dem Meißner Dom, 2014.

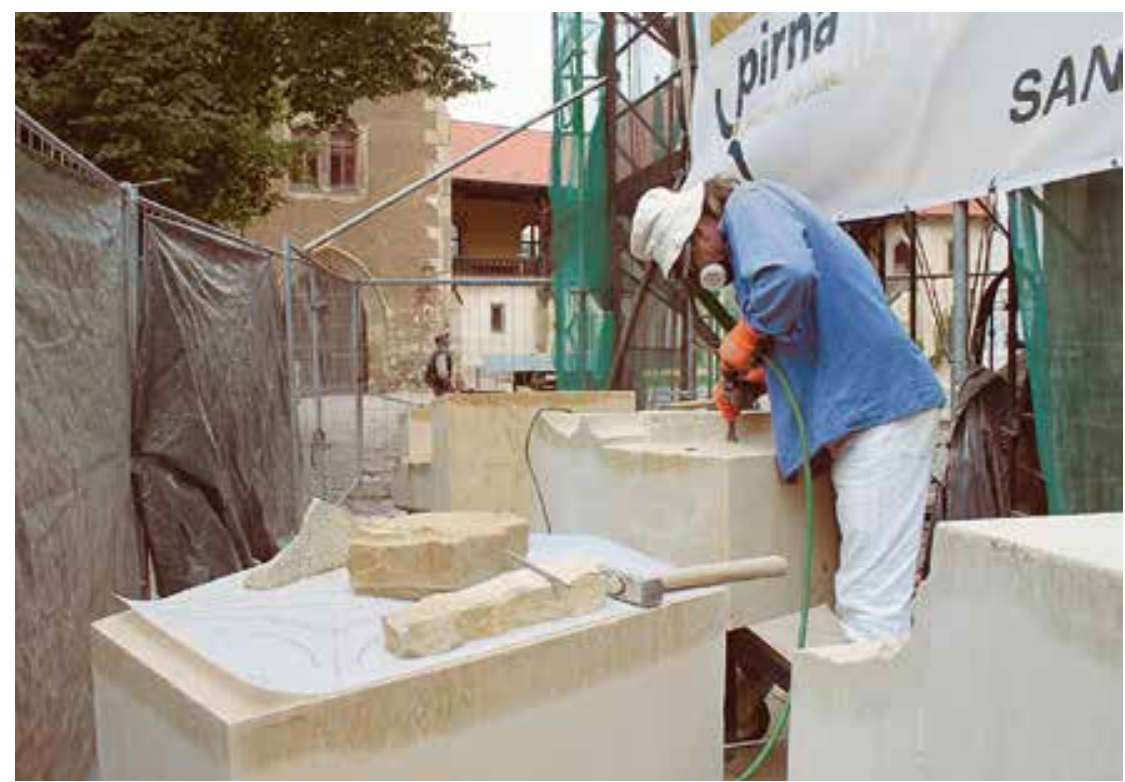




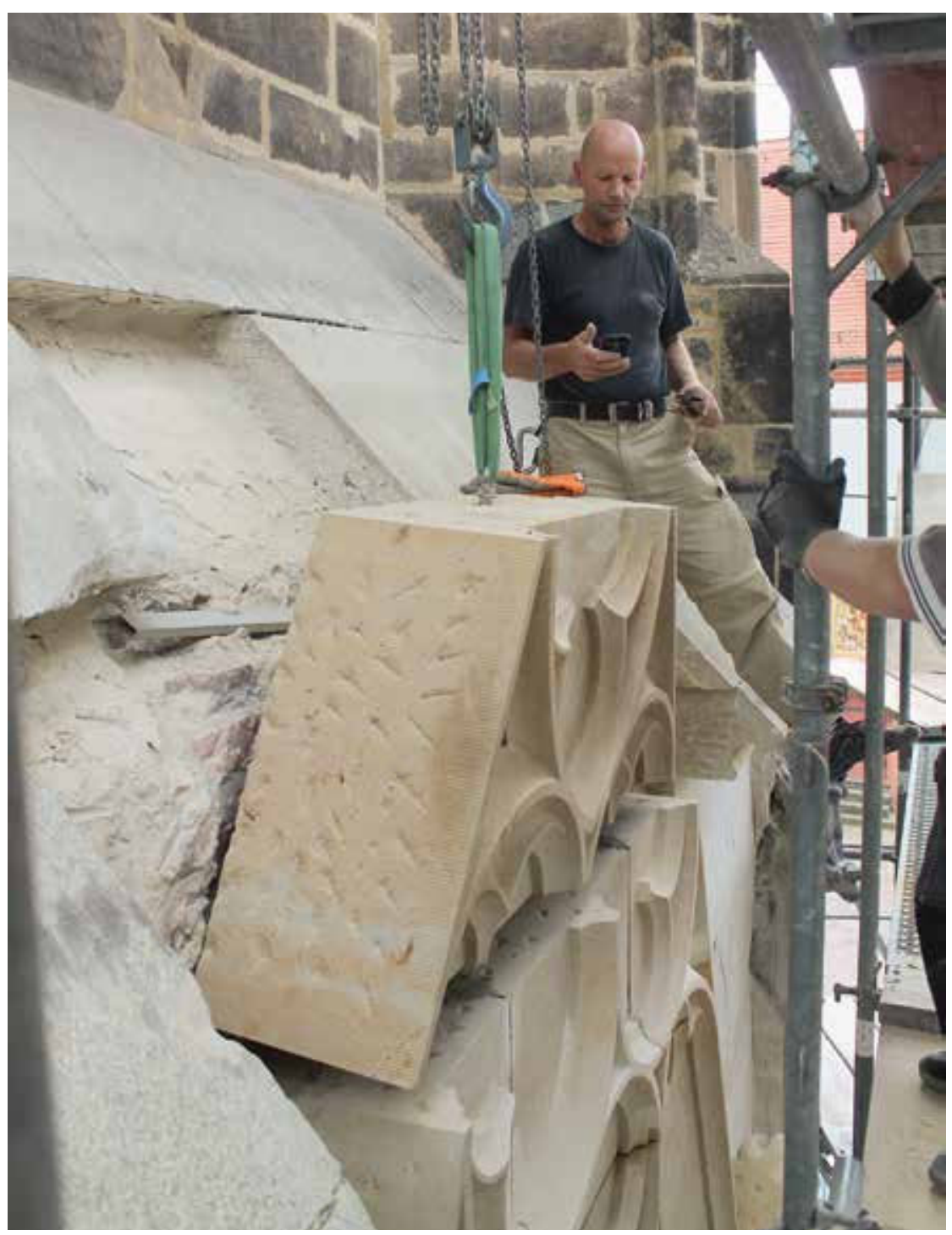

Das Aufsetzen eines Werksteins des Wimperges am Portal des Meißner Domes, 2014.

7 Magirius, Heinrich: Stil und Stilkorrekturen in der Denkmalpflege seit 1900. Annäherungen an das rechte $\mathrm{Maß}$, in: Monumenta Misnensia, Jahrbuch für Dom und Albrechtsburg zu Meißen 11 (2013/2014), S. 48 . erische Vorlagen, die weiteren Steinmetz- und Bildhauerarbeiten sowie die komplizierte Aufgabe der Logistik der Werksteinbereitstellung und des Steinversatzes.

\section{Die Wimperg-Baustelle}

Seit dem Frühjahr 2014 „zierte“ ein durch die Dombauhütte gestelltes Gerüst die WimpergBaustelle und die als Gerüstbespannung nahezu auf den Maßstab 1:1 vergrößerte Zeichnung des Wimpergs machte den Besuchern deutlich, was künftig hinter den Planen und Bauzäunen im Entstehen war. Dazu richtete man eine temporäre Steinmetz-Bauhütte ein, damit ein Teil der Werksteine ganz traditionell vor Ort bearbeitet werden konnte.

Um den neuen Wimperg einbauen zu können, musste natürlich die Hartung'sche Vormauerung aus Sandstein weichen. Schon beim Ausbau der ersten Steinquader aus der Dreiecksfläche kamen Baubefunde zum Vorschein, welche die Interpretation der Ergebnisse der Voruntersuchungen noch einmal bestätigten: Eine sich durch kleinteilige Mauerstrukturen abzeichnende dreieckige Fläche einer Ausmauerung ließ vermuten, dass hier bereits einmal ein Giebel, zumindest aber ein dreieckiges Gebilde gestanden haben musste.

Hartungs Eingriffe hatten allerdings die historische Werksteinoberfläche und somit auch alle Befunde unwiederbringlich zerstört. Zudem hatte er die Werksteine mit einem Zementmörtel vermauert und vergossen, deren Abbruch einen enormen Kraftaufwand und Technikeinsatz erforderte. Deshalb wurde entschieden, nur jede zweite Steinlage der Werksteine des neuen Wimpergs tief einzubinden und jede dritte Lage durch Edelstahlanker rückseitig zu verhängen.

Bis Mitte September 2014 waren die Steinmetz- und Bildhauerarbeiten soweit vorangeschritten, dass mit dem Versatz der Werksteine begonnen werden konnte. Bis Oktober waren die Werksteine des gesamten Wimperggiebels soweit versetzt, dass das Aufsetzen der Kreuzblume vorbereitet werden konnte. Zuvor waren jedoch noch die Übergänge der einzelnen Werksteine anzuarbeiten und den Oberflächen im Sinne einer Vereinheitlichung der unterschiedlichen "Steinmetzhandschriften" ein einigermaßen gleiches Scharrierbild (bunter Hieb) zu verleihen. Für die baustatische Festigkeit sorgte ein satteldachartiger Mauerwerkskörper, der rückseitig hinter der Giebelfläche aus normalformatigen Hartbrandsteinen aufgemauert wurde. Der Mauerwerkskörper wurde anschließend ausgefugt und eine glatte Oberfläche vorbereitet, auf der später mühelos die Bleiverdachung aufgelegt werden konnte.

Auch wenn der wichtigste Bauabschnitt geschafft war, so galt es nun, noch viele Details baulich zu lösen. Nach dem Einbau der beiden Wasserspeier waren es vor allem die beiden Wandflächen links und rechts des Wimperggiebels, die jetzt zurückgearbeitet werden mussten. Diese räumliche Durchbildung war mit Hilfe der 3D-Darstellung hervorragend herausgearbeitet worden. Das Maß der alten Wandvorderkante der Arnold'schen Wimperglösung hatte Hartung bei seinem Umbau 1909/10 insofern dokumentiert, da er seine neue Wandkonstruktion im oberen Bereich seitlich mit einer tiefen Nische an die mit Blendmaßwerken verzierte Wasserspeierflanken anschloss, während im unteren Bereich die Wand ohne Nische ausgebildet und das Blendmaßwerk am Strebepfeiler fast völlig verdeckt war. Die gesamte Wandfläche abzubauen und die Arnold'sche Mauer freizulegen, wäre zwar 
technisch möglich gewesen, aber wegen der $\mathrm{zu}$ erwartenden Zerstörungen an der originalen Quaderoberfläche des Arnold'schen Mauerwerks wenig sinnvoll gewesen. So wurde entschieden, das Hartung-Mauerwerk so weit zurückzuarbeiten, dass es der alten Position sehr nahe kommen würde und wie eine „Folie“ vor dem Arnold'schen Original bestehen bleibt. Diese neue Sichtfläche wurde vom Steinmetz glatt abgearbeitet; alle UnregelmäBigkeiten wurden im Bestand belassen, um somit die ältere Zeitschicht gegenüber der jüngsten Zeitschicht, dem Wimperggiebel, zu dokumentieren.

Mit dem Zurückarbeiten der Vorderseite um $15 \mathrm{~cm}$ musste auch das profilierte Gesims abgebaut werden, da ja nun die neue Gesimskante wesentlich weiter nach oben gerückt war. Mit der Entscheidung, die pultdachartigen Steinflächen hinter der Front und links und rechts vom Giebel mit Walzblei abzudecken, konnte eine neue Gesimsausbildung entfallen. Stattdessen wird das Regenwasser hier durch eine in der Dachfläche liegende und somit optisch versteckte Kastenrinne aus Walzblei aufgefangen und darin den Wasserspeiern zugeleitete. Diese „speien“ nun das bauschädliche Regenwasser weit vom Gebäude weg. Damit stellt die neue Konstruktion aus bautechnischer Sicht eine wesentliche Verbesserung gegenüber der bisherigen Lösung dar, ohne dass diese Änderung gegenüber dem Original optisch wahrgenommen wird.
Am 4. November 2014 konnte die Kreuzblume aufgesetzt werden. Bis zum Jahresende 2014 folgten noch kleinere Arbeiten. Heute präsentiert sich nun der Wimperg über dem Westportal in hellem Sandstein. Es wird nun einige Jahre dauern, bis sich die neue Baukonstruktion durch die natürliche Patinierung ihrer Umgebung angepasst hat.

Heinrich Magirius stellte zusammenfassend fest: „Bei aller Achtung vor dem, Vater' der sächsischen Denkmalpflege, Cornelius Gurlitt, können wir heute kaum mehr verstehen, warum er und seine Kommission den neugotischen Wimperg über dem Westportal des Meißner Domes, hässlich' fanden. Im Gegenteil: Der Wimperg war eine ,stilgemäße' Antwort auf Veränderungen des 17. Jahrhunderts, die dem Dom wahrlich nicht gut $z u$ Gesicht standen. Seine Wiederherstellung im 21. Jahrhundert wird - so hoffen wir - eines Tages als selbstverständlicher Bestandteil des alten, immer wieder erneuerten Domes erscheinen"?

Die Rekonstruktion des Wimpergs über dem Westportal der Fürstenkapelle des Meißner Doms wurde mit dem Peter-Parler-Preis 2015, Sonderpreis für Dombauhütten, ausgezeichnet. Der von der Deutschen Stiftung Denkmalschutz und von dem Bundesverband Deutscher Steinmetze ausgelobte Preis wird alle zwei Jahre verliehen. Die Preisverleihung an Dombaumeister Günter Donath erfolgte am 14. Mai 2015 in Nürnberg.

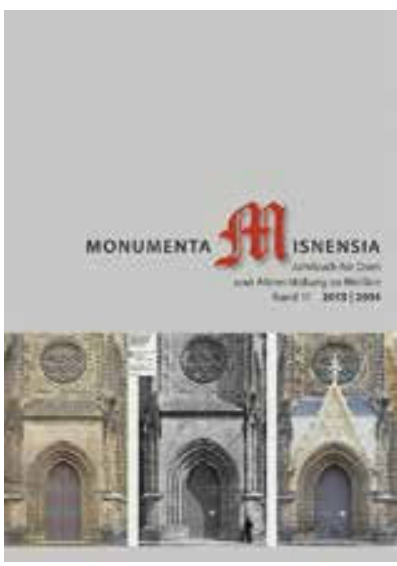

Ausführliche Informationen zur Rekonstruktion des Wimpergs sind in Monumenta Misnensia, Jahrbuch für Dom und Albrechtsburg zu Meißen. 11 (2013/2014) zu finden. Der Band (112 Seiten, 9,80€) kann bestellt werden bei:

\section{Hochstift Meißen}

Domplatz 7

01662 Meißen

Tel.: 03521-452400

E-Mail:Info@dom-zu-meissen.de.

\section{Autor}

Dombaumeister

Günter Donath

Wilsdruff

\section{Subskriptionsangebot}

Die Redaktions- und Verlagsgesellschaft Elbland mbH aus Meißen bietet zwei Bücher aus der Reihe „Adel in Sachsen“ zur Subskription an. Beide Bücher sind umfangreiche Kompendien zur Adelsgeschichte Sachsens und zugleich sozialgeschichtliche Studien zum Lebensalltag des niederen Adels.

Matthias Donath: Schwarz und Gold. Die Familie von Watzdorf in Thüringen, Sachsen und Schlesien

Ca. 550 Seiten mit zahlreichen Abbildungen und Karten, fester Einband, $17,5 \times 24,5 \mathrm{~cm}, 1$. Auflage, erscheint voraussichtlich im Juli 2015, Preis: 29,90 Euro. Subskriptionspreis bei Bestellung bis 31. Mai 2015: 25,00 Euro

\author{
Matthias Donath: Rotgrüne Löwen. \\ Die Familie von Schönberg \\ in Sachsen \\ 640 Seiten mit zahlreichen \\ Abbildungen und Karten, fester Einband, \\ $17,5 \times 24,5 \mathrm{~cm}, 2$. Auflage \\ (die 1. Auflage war kurz nach \\ Erscheinen vergriffen), Preis: 29,90 Euro. \\ Subskriptionspreis bei Bestellung \\ bis 31. Mai 2015: 25,00 Euro
}

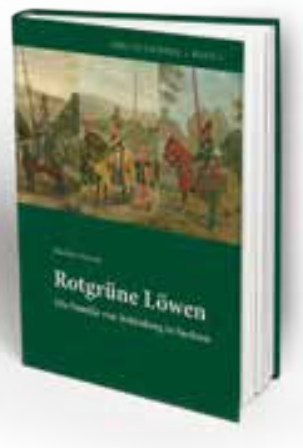

